\title{
The Navigated Image Viewer - Evaluation in Maxillofacial Surgery
}

\author{
S. Weber, M. Klein, A. Hein, T. Krueger, T.C. Lueth, and J. Bier \\ Berlin Center for Mechatronical Medical Devices \\ Department Maxillofacial Surgery - Clinical Navigation and Robotics \\ Prof. Dr. mult. h.c. Juergen Bier and Prof. Dr. Tim C Lueth \\ Medical Faculty Charité - Humboldt University at Berlin \\ Campus Virchow, Augustenburger Platz 1, 13353 Berlin, Germany \\ \{stefan.weber, martin.klein, andreas.hein, \\ timo.krueger, tim.lueth, juergen.bier\}@charite.de \\ http://www. srl-berlin.de
}

\begin{abstract}
In this paper first results in clinical evaluation of a new mobile image viewing device are described. The system comprises a compact and mobile viewing device that can be tracked by an optical position sensor. It is capable of displaying reasonable parts of a medical image data set (i.e. CT) on its screen in relation to the spatial position regarding a patient. This article describes first results and application details in applying this viewing device to clinical indications in maxillofacial surgery.
\end{abstract}

\section{Introduction}

To support surgeons during medical interventions, a navigated and mobile viewing device - NaviView - for computer tomographical imaging data has been developed. This imaging device displays medical imaging data near the patient and in spatial correlation to the patient.

The approach comprises a 6.4"-TFT-LCD screen that is spatially tracked using a standard optical tracking system (Polaris, NDI Canada). Therefore, a passive tracking device is attached to the housing for measuring the position of the device in space. A standard personal computer is used as a control unit. NaviView is connected to the video output of the control unit. This mobile and navigated setup allows for viewing of medical image data directly at the patient and within the sterile area during a surgical intervention. Given the relative spatial position and orientation between the NaviView system and the patient, the control unit renders an reasonable part of the medical image data to the display. This allows intuitive viewing of image data sets from arbitrary positions and orientations relative to the patient. The compact display, consisting of a CCD-camera, a LCD-screen and a touch screen sensor is only $270 \mathrm{x}$ $200 \times 50 \mathrm{~mm}$, weighting around gr. 500. This compactness allows for application even within the surgical situs. To control the device, the screen is equipped with a resistive touch sensor. Using the touch screen the surgeon can simply navigate through the scene, zoom the image or display different interesting structures and change their appearance and further imaging parameters. 
This paper deals with issues of clinical application and experiences in integrating this system into the surgical process. It will be shown that the application of a such a navigated viewing device is extremely helpful for interpreting medical imaging data such as CT images. Based upon this system a number of implemented visualization modes and their support to the surgeon is discussed.

\section{State of the Art}

A number of different technical approaches for visualization of medical imaging are described in literature:

Stationary computer screens and print outs: Medical images are most commonly viewed and interpreted using colour calibrated computer screens. Intra-operatively, surgeons rely on print outs that are viewed in front of an illuminated screen. Interpretation becomes difficult when the surgeon has to walk back and for between the viewing screen and the surgical scene.

Surgical Microscopes: are used to augment imaging data from different modalities onto the viewing area of the microscope [1][2]. However, such systems are too large, expensive and cumbersome to manipulate for most short lasting intraoperative inspection of medical images. Such microscope systems are seldom found outside neuro- and maxillofacial surgery departments and seem to be not available to medical staff outside of specialised hospitals.

Head-Mounted Displays: Several research projects cover different aspects of viewing medical imaging data using head mounted displays (HMD) [3][4]. Although it seems intuitive to use HMD's, surgeons complain about the viewing handicaps for their occluded views and the weight of the systems to be worn on the head during an intervention. Handling unsterile HMD's by the surgeons seems rather difficult.

Translucent Displays: Translucent displays are inserted into the surgeons field of view and different imaging modalities can be augmented on the screen [5][6]. Being a powerful visualization method, the technical setup is rather difficult, since the viewers (i.e. the surgeons) eyes need to be tracked in space to align the computer created image with the real view of the surgical scene.

The drawbacks of the above mentioned systems regarding cost effectiveness, handling in a surgical scene, sterilization issues and technical complexity have lead to the development of a new navigated image viewer. Advantage of the NaviView system is a direct projection of the correlation between medical imaging data and a real scene on a compact, portable and flexible to handle miniature screen in close distance to a patients body. The surgeon may not be disturbed by head mounted displays or other extensive and cumbersome to handle equipment. The proposed and developed device is inexpensive and easy to integrate in clinical environments. The system and its capability to augment medical imaging modalities onto a video modality of the same patient are described in detail in [7]. 


\section{Description of Application}

The following chapters describe technical and clinical details of the first application of this novel device in maxillofacial surgery. Besides an introduction into the systems mode of operation, the actual clinical indication, the workflow of application is depicted and the benefit for the surgeons will be discussed.

\subsection{The Navigated Image Viewer}

The navigated viewing device comprises a mobile and compact flat panel display and a localizer system for spatial tracking (Fig. 1).

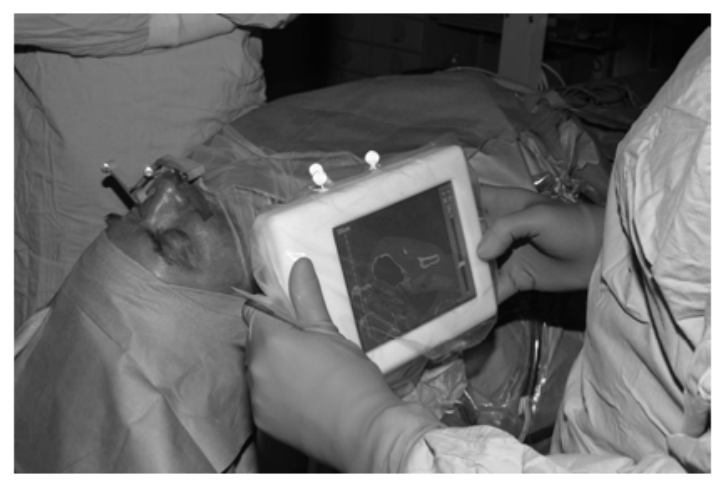

Fig. 1. The navigated image viewer is a mobile computer screen with an optical localizer system and a video camera integrated to sensor the back side of the viewer (left). Pre- and intraoperative phases for using a navigated image viewing device. The preoperative phase consists of preparation of a dental splint and image acquisition. During the intervention the system is used for displaying imaging modalities (right).

The image viewer is attached to a navigation platform (RoboDent $\mathrm{GmbH}$, Berlin/Germany) that consists of a processing unit and the optical tracking system Polaris (NDI, Ontario/Canada). The tracking system cam measures the position and orientation of NaviView disp and the patient pat (Fig. 2) to form the transformation:

$$
{ }^{\text {pat }} \mathbf{T}_{\text {disp }}=\left({ }^{\mathrm{cam}} \mathbf{T}_{\text {pat }}\right)^{-1} \cdot{ }^{\mathrm{cam}} \mathbf{T}_{\text {disp }}
$$

The image data set is registered to a set of markers mar and from there to the patients tracking device pat using:

$$
{ }^{\text {pat }} \mathbf{T}_{\text {ima }}={ }^{\text {pat }} \mathbf{T}_{\text {mar }}{ }^{\text {mar }} \mathbf{T}_{\text {ima }}
$$

whereby ${ }^{\text {pat }} \mathbf{T}_{\text {mar }}$ is the reproducible position of the tracking device to the markers reference frame. The position of the markers are automatically detected in the image data set, described in [8], and a coordinate transformation ${ }^{\text {mar }} \mathbf{T}_{\text {ima }}$ is created. A transformation between the tracking device and the viewing screen of the viewer is described by ${ }^{\text {view }} \mathbf{T}_{\text {disp }}$. The spatial relationship between the image data and a position on the viewing screen can then be described by: 


$$
{ }^{\text {view }} \mathbf{T}_{\text {ima }}={ }^{\text {view }} \mathbf{T}_{\text {disp }}{ }^{\text {disp }} \mathbf{T}_{\text {world }} \cdot\left({ }^{\text {pat }} \mathbf{T}_{\text {world }}\right)^{-1} \cdot{ }^{\text {pat }} \mathbf{T}_{\text {ima }}
$$

The complete transformation of the screen's corner points ${ }^{\text {view }} \mathbf{p}_{\mathrm{ul}}$, ${ }^{\text {view }} \mathbf{p}_{\mathrm{ur}}$ and ${ }^{\text {view }} \mathbf{p}_{\mathrm{ll}}$ (upper left and right and lower left corner) into the image coordinate system such as:

$$
{ }^{\text {ima }} \mathbf{p}_{\text {ul }}=\left({ }^{\text {vis }} \mathbf{T}_{\text {ima }}\right)^{-1} \cdot{ }^{\text {view }} \mathbf{p}_{\text {ul }}
$$

a visualization plane vis in the distance $d_{\text {view }}$ can be described and the voxel values of the image data from within this plane are linearly transferred to the viewing screen.

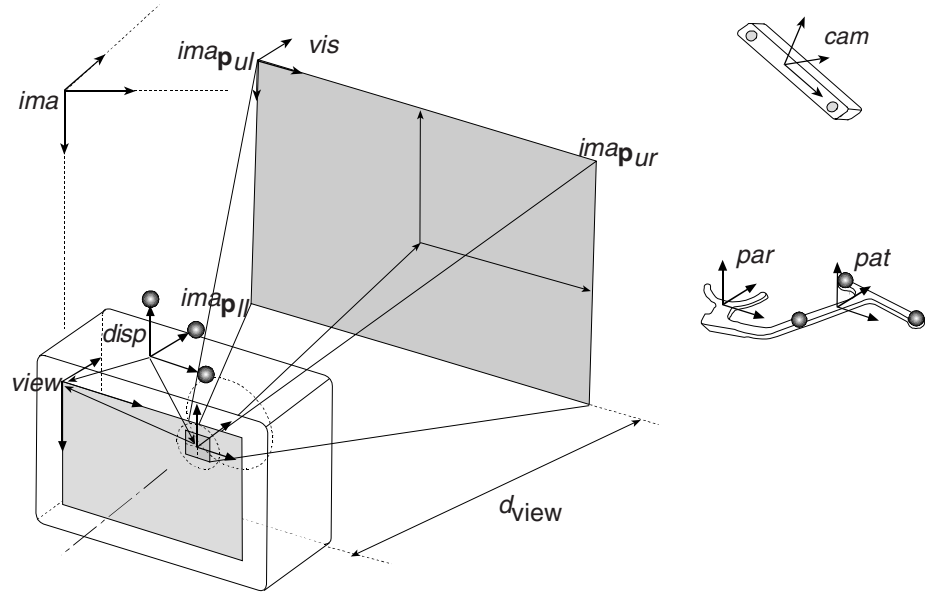

Fig. 2. Graphical model to visualize a specific plane from within a three dimensional image data set. The visualization plane correlates with NaviViews position relative to the patient and therefore to the image data.

This model allows for intuitive projection of image data. By changing the viewing parameters such as $d_{\text {view }}$ different visualization modes can be realized. To optimally interpret the visualized images the surgeon can choose from different visualization modes:

- Fixed distance mode: The images being displayed are created in a fixed distance in front of the NaviView. To visualize images in different depths within the patient, the surgeon moves the system along the viewing axis. A slider element within the graphical user interface (touch screen) serves for selection of appropriate zoom factors in a way that the surgeon can view any actual image situation from a landscape zoom to a microscope zoom view.

- Fixed Focus mode: The fixed focus mode can be applied, once a designated internal structure is of interest to the surgeon. Moving the NaviView in a way, that the interesting structures is centred within the image and than activating the fix focus mode allows to view the focused area from different positions and angles. NaviView calculates the appropriate viewing plane within the image data set according to the actual spatial relationship between the patient and the viewing device. 
To operate the system and to change viewing parameters a graphical user interface is integrated, that can be operated via touch screen. It consists of a simple, yet intuitive to operate number of graphical elements. Importantly, the medical images are displayed on the screen and part of it is reserved for displaying the operating elements. The user finds status information such as visibility states and visualization modes (Fig. 3).

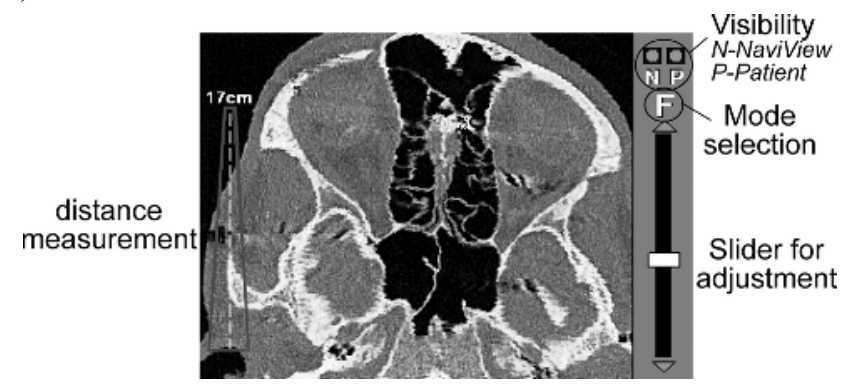

Fig. 3. The user interface comprises a touch screen and a set of easy to understand graphical gadgets.

A graphical element depicts the current viewing distance between the system and the viewing plane. A slider element is placed for intuitive adaptation of visualization parameters such as zoom factor or viewing distance. However, the functionally minimal user interface does not overstrain the surgeon and still provides for maximum interpretability of the medical image data.

\subsection{Clinical Indication}

To discuss the advantages and benefits of using navigated imaging in maxillofacial surgery, a typical clinical indication, familiar to maxillofacial and trauma surgeons, has been chosen, to apply the NaviView-System. A male person delivered to the hospital with a typical zygoma fracture became the first patient for applying this novel idea and system for image viewing.

A zygoma fracture is a high dimensional fracture of bone structures within the midfacial area (Fig. 4 a). Within this anatomical area, a number of important internal structures such as the orbital floor are affected due to the fracture. These structures are important for correct vision and serve several other aesthetic and functionally important aspects. The optimal outcome of a surgical treatment of zygoma fracture is a correct three dimensional repositioning of the dislocated bone segments to cope for the above mentioned reasons. To understand the clinical situation being treated, the surgeon has to determine the number and spatial relationships of all fractured parts of the zygoma and the connecting structures. Since fracture lines most often lay within different planes of the image set, the surgeon finds it difficult to understand the complex situation.

A correct and optimal interpretation of available imaging modalities such as CT is imperative in order to understand the complex three dimensional situation and to 
identify possible problems, that could possibly be encountered during the surgical intervention.

\subsection{Image Acquisition and Registration}

A CT scan (Siemens Somatom, Germany) of the patients head was performed and the imaging data exported to the navigated image viewer using DICOM 3.0 Standard. A dental splint was produced using a negative imprint taken from the patients upper jaw at the beginning of the treatment. A navigation bow with a set of titanium markers is attached to the splint. The splint/navigation bow device is clipped into the patients upper jaw during image acquisition and during the surgery (Fig. 4 b).
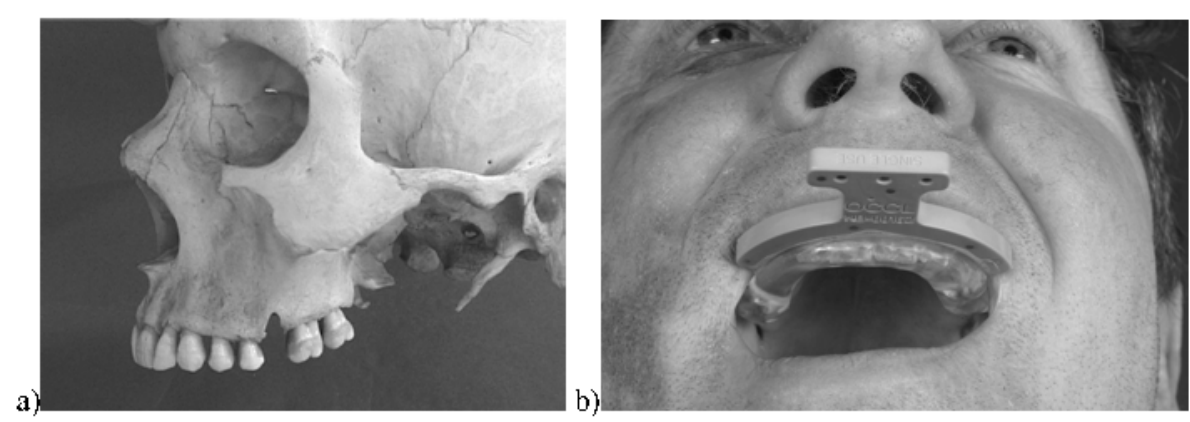

Fig. 4. a) Typical fracture lines of a zygoma in the midfacial area [9]. b) The registration device consists of a dental splint and a navigation bow, worn on the patients upper jaw. The device is attached to the patient during image acquisition and the viewing phase.

Part of the preparation phase is an automatic detection process of the titanium markers in the navigation bow as described in [8]. The marker positions within the medical images and their known and reproducible position relative to the attached patient localizer enables a safe, precise and easy to use registration.

\subsection{Intraoperative Application of the Navigated Image Viewer}

NaviView is ready to use after it has been prepared for the sterile environment using sterile drapes. The imaging data set is loaded into the system. As soon as both the patients and NaviViews localizer are visible to the position sensor and the system is in a useful spatial correlation to the patient, imaging data is displayed to the surgeon (Fig. 3 right). The user moves the NaviView system freely around the interesting areas and interprets the images being displayed. The two dimensional images depicted are selected by spatially moving the system. A direct hand-eye-coordination can be established due to visual feedback given to the surgeon while changing NaviViews position in space. 

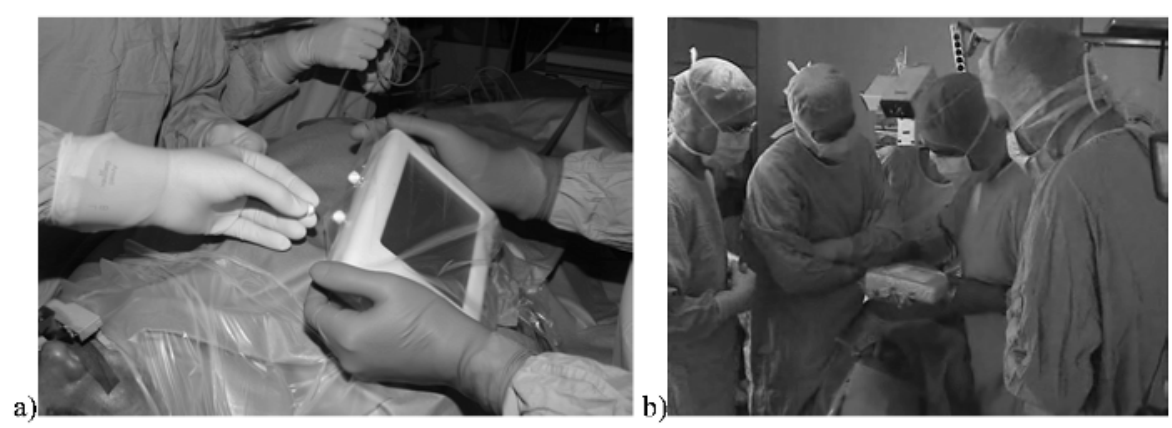

Fig. 5. a) The navigated image viewer is covered in sterile drapes to be used directly at the surgical area. Sterile reflectors are attached to the outside for optical tracking. b) NaviView was used by several surgeons in parallel to extensively discuss the clinical situation before the actual surgical intervention.

\section{Results and Conclusion}

The preclinical evaluation phase consisted of numerous consultations of the developing team and the clinical users to find an optimal visualization of the high dimensional image data in a simple and optimal to understand manner.

Surgeons reported, that using the fixed distance mode gives them most intuitive understandability due to the direct visual feedback created from the specific spatial situation.

Applying the NaviView system provided the surgeons with interesting insights into the specific clinical situation. Dynamic visualization in a close spatial context to the patient has been proven to be extremely helpful in understanding the spatial relationship of the clinical situation. The depicted situation requires a sophisticated understanding of the several fracture lines within the bone segments and the spatial position of the fractured element. This is of great importance for the later surgical treatment, as to find an optimal initial insertion position for the surgical tools.

Despite different initial expectations we found that surgeons rather rely on ordinary two dimensional images rather than sophisticated 3D graphic renderings. Their expertise in interpreting 2D images is rather large, since this form of visualization is the current clinical standard. Nevertheless, there might be interesting and real helpful forms of $3 \mathrm{D}$ visualization that need to be found in future.

NaviView has been found to be a simple to use, easy and inexpensive to integrate and powerful image visualization device. The successful clinical application of this novel imaging device in maxillofacial surgery supplied us with interesting aspects for further research in optimal clinical integration as well as ideas for applying NaviView in different clinical fields such as trauma and orthopaedic surgery. Great potential lies in the research of intuitive and helpful visualization modes to support medical staff during the interpretation phase. Another aspect of future work lies in integrating other medical imaging modalities into the system and provide surgeons with new perspectives of image data. 
Acknowledgement. This research work has been performed at the Department for Maxillofacial Surgery - Clinical Navigation and Robotics, Prof. Dr. Dr. Juergen Bier and Prof. Dr. Tim C. Lueth, Medical Faculty Charité, Humboldt-University Berlin and the Fraunhofer-Institut für Produktionsanlagen und Konstruktionstechnik - IPK Berlin, Prof. Dr.-Ing. Eckart Uhlmann. The work has been supported by the Alfried Krupp von Bohlen und Halbach-Stiftung. Parts of the research have been supported financially by the European Regional Development Fund (ERDF), Deutsche Krebshilfe (granted to Prof. Dr. Dr. J. Bier, PD Dr. P. Wust) and the Berliner Sparkassenstiftung Medizin (granted to Prof. Dr. T. Lueth, Dr. Dr. Ernst Heissler, Prof. Dr. Dr. Berthold Hell). Special thanks to Marc Kneissler and Dirk Schauer for their engaging support in designing and constructing of NaviView, Daniel Szymanski for his valuable software modules and Ingolf Karst for his soldering craftsmanship. Special thanks to the companies RoboDent, Altatec, Ziehm Instrumentarium, Planmeca, Straumann, Medtronic, Philips and Locke Dentaltechnik for their support of the project.

\section{References}

[1] Hill DLG, Hawkes DJ, Fenlon MR, Strong AJ, Cox TCS, Gleeson MJ (2000) "Stereo augmented reality in the surgical microscope." Presence: Teleoperators and Virtual Environments, 9(4):360-368, 2000

[2] Birkfellner W., Figl M., Huber K., Hummel J., Hanel R., Homolka P., Watzinger F., Wanschitz F., Ewers R. and Bergmann H. (2001): Calibration of projection parameters in a head mounted display for augmented reality visualization in image guided therapy. SPIE Medical Imaging, 2001.

[3] Fuchs H., Mark A. Livingston, Ramesh Raskar, D'nardo Colucci, Kurtis Keller, Andrei State, Jessica R. Crawford, Paul Rademacher, Samuel H. Drake, and Anthony A. Meyer, MD.(1998):Augmented Reality Visualization for Laparoscopic Surgery., Proceedings of First International Conference on Medical Image Computing and Computer-Assisted Intervention (MICCAI '98), Cambridge, MA, USA., October

[4] Nomad Display, Microvision Inc. U.S.A. www.mvis.com

[5] Schnaider M. (2003): Medarpa - A Medical Augmented Reality System for MinimalInvasive Interventions, Medicine meets Virtual Reality (MMVR), Newport Beach, California/USA, 22-25 January 2003.

[6] G. Stetten, V. Chib, R. Tamburo, " Tomographic Reflection to Merge Ultrasound Images with Direct Vision," IEEE Proceedings of the Applied Imagery Pattern Recognition (AIPR) annual workshop, 2000, pp. 200-205.

[7] Weber S., Hein A., Lueth T. C. (2002): A Mobile and Navigated Image Viewer for Surgery - Evaluation of Precision. Medicine meets Virtual Reality (MMVR), Newport Beach, California/USA, 22-25 January 2003.

[8] Schermeier, O.; T. Lueth, J. Glagau, D. Szymanski, R. Tita, D. Hildebrand, M. Klein, K. Nelson, J. Bier (2001): Automatic patient registration in computer assisted maxillofacial surgery. Medicine Meets Virtual Reality 2002, Newport Beach, California/USA, 23-26 January 2002.

[9] Dannhauer, K.-H.(2003): Webpage from www.uni-leipzig.de/ kfo/ GNE/theotext.htm, $20^{\text {th }}$ of March 2003 